\begin{tabular}{|c|c|}
\hline Title & Evidence for the Diffusion of A u A toms into the Te UPD Lay er Formed on a A u(111) Substrate \\
\hline Author(s) & $\begin{array}{l}\text { Kawamura, Hiroy uki; Takahasi, Masamitu; Hojo, Nobuhiko; Miyake, Masao; Murase, Kuniaki; Tamura, Kazuhisa; } \\
\text { U osaki, Kohei; A wakura, Y asuhiro; Mizuki, Jun'ichiro; Matsubara, Eiichiro }\end{array}$ \\
\hline Citation & $\begin{array}{l}\text { Journal of The Electrochemical Society, 149(2), c83-c88 } \\
\text { https://doi.org/10.1149/1.1429928 }\end{array}$ \\
\hline Issue Date & 2002 \\
\hline Doc URL & http:/hdl.handle.net/2115/50232 \\
\hline Rights & $\begin{array}{l}\text { (C) The Electrochemical Society, Inc. 2001. All rights reserved. Except as provided under U.S. copyright law, this work } \\
\text { may not be reproduced, resold, distributed, or modified without the express permission of The Electrochemical Society } \\
\text { (ECS). The archival version of this work was published in J. Electrochem. Soc. } 2002 \text { volume 149, issue 2, C83-C88. }\end{array}$ \\
\hline Type & article \\
\hline File Information & JES149-2_C83-C88.pdf \\
\hline
\end{tabular}

Instructions for use 


\section{Evidence for the Diffusion of Au Atoms into the Te UPD Layer Formed on a Au(111) Substrate}

Hiroyuki Kawamura, Masamitu Takahasi, Nobuhiko Hojo, Masao Miyake, Kuniaki Murase, Kazuhisa Tamura, Kohei Uosaki, Yasuhiro Awakura, Jun'ichiro Mizuki and Eiichiro Matsubara

J. Electrochem. Soc. 2002, Volume 149, Issue 2, Pages C83-C88. doi: 10.1149/1.1429928

Email alerting service box at the top right corner of the article or click here

To subscribe to Journal of The Electrochemical Society go to: http://jes.ecsdl.org/subscriptions 


\title{
Evidence for the Diffusion of Au Atoms into the Te UPD Layer Formed on a $\mathrm{Au}(111)$ Substrate
}

\author{
Hiroyuki Kawamura, ${ }^{a, z}$ Masamitu Takahasi, ${ }^{a}$ Nobuhiko Hojo, ${ }^{b}$ Masao Miyake, \\ Kuniaki Murase, ${ }^{\mathrm{b}, *}$ Kazuhisa Tamura, ${ }^{\mathrm{c}}$ Kohei Uosaki, ${ }^{\mathrm{c}, *}$ Yasuhiro Awakura, ${ }^{\mathrm{b}}$ \\ Jun'ichiro Mizuki, and Eiichiro Matsubara ${ }^{\mathrm{d}}$ \\ ${ }^{a} J a p a n$ Atomic Energy Research Institute, Synchrotron Radiation Research Center, Kansai Research \\ Establishment, Hyogo 679-5148, Japan \\ ${ }^{b}$ Department of Materials Science and Engineering, Kyoto University, Kyoto 606-8501, Japan \\ ${ }^{c}$ Physical Chemistry Laboratory, Division of Chemistry, Graduate School of Science, Hokkaido University, \\ Sapporo 060-0810, Japan \\ 'Institute for Materials Research, Tohoku University, Sendai 980-8577, Japan
}

\begin{abstract}
The structure of a Te layer formed on a Au(111) substrate by underpotential deposition (UPD) in an electrolytic solution has been studied using in situ surface X-ray diffraction technique. The measurements were carried out for a series of samples which were kept at UPD potential for 4 to $59 \mathrm{~h}$. The results revealed that the Te UPD layer is unstable. The top layer is analyzed to consist of the UPD Te atoms and $\mathrm{Au}$ atoms which diffuse from the Au(111) substrate. Also, the Te UPD layer does not have the structure with periodicity reported in previous works, such as $(\sqrt{3} \times \sqrt{3}) \mathrm{R} 30^{\circ}$ after ample time elapses. Stripping voltammetry for the Te UPD layer shows that the interaction between Te and Au increases with time, supporting the finding that the top layer is a mixture of $\mathrm{Te}$ and $\mathrm{Au}$.
\end{abstract}

(C) 2001 The Electrochemical Society. [DOI: 10.1149/1.1429928] All rights reserved.

Manuscript submitted January 4, 2001; revised manuscript received September 12, 2001. Available electronically December 20, 2001.

A single atomic layer can be formed by the electrochemical processes using underpotential deposition (UPD), which is a surfacelimited reaction to a submonolayer or monolayer coverage. In UPD, the electrochemical deposition of foreign metals onto substrate is performed at a positive potential relative to the reversible Nernst potential for bulk deposition. The method which alternates the UPD of two different elements to form a binary compound is referred to as the electrochemical atomic layer epitaxy (ECALE). ${ }^{1}$ In ECALE, the electrochemical potential is used to restrict the deposition of constituent elements through UPD. It can be considered as the electrochemical analog of the atomic layer epitaxy. Since an ideal atomic layer epitaxy can be achieved by ECALE, this method is promising for creating new materials and devices.

Formation of cadmium chalcogenide layers such as $\mathrm{CdTe}$ on $\mathrm{Au}$ single-crystal substrate using the ECALE technique has been investigated from the viewpoint of their applications to solar cells. ${ }^{2-4}$ Since the deposition of $\mathrm{Cd}$ on a $\mathrm{Au}$ substrate easily produces intermetallic compounds, such as $\mathrm{Cd}_{3} \mathrm{Au},{ }^{5}$ the first step to the formation of CdTe films by ECALE is not the reductive UPD of Cd. Rather, Te is first deposited by UPD on a Au single-crystal substrate. However, the reductive Te UPD on Au single-crystal substrate is a complex process. In cyclic voltammetry studies, two cathodic peaks have been observed prior to the peak of the bulk deposition. ${ }^{6-9}$ The nobler peak is referred to as the first UPD and the other as the second UPD. Due to the irreversible nature of the $\mathrm{Te} / \mathrm{Te}$ (IV) redox, only the first UPD occurs at a potential which is positive with respect to the equilibrium potential of bulk Te deposition, the latter being calculated from thermodynamic data. Therefore, the first UPD is the true underpotential deposition and is referred to as UPD in this paper.

The structure of Te UPD layer formed on a $\mathrm{Au}(111)$ substrate has been studied using scanning tunneling microscopy (STM) and atomic force microscopy (AFM) ${ }^{6-8}$ There seems to be a controversy on the structure of the Te UPD layer, i.e., an AFM study reported a $(\sqrt{3} \times \sqrt{3}) \mathrm{R}^{\circ} 0^{\circ}$ structure, $^{8}$ and STM studies reported a $(12 \times 12)$ structure. ${ }^{6,7}$ The discrepancies in the literature were recently discussed from an STM study, proposing the structures that the $(\sqrt{3}$ $\times \sqrt{3}) \mathrm{R} 30^{\circ}$ and $(12 \times 12)$ periodicity was accounted for by the individual atoms with $\mathrm{a}(\sqrt{3} \times \sqrt{3}) \mathrm{R} 30^{\circ}$ and the domain wall structure with a $(13 \times 13)$ periodicity, respectively. ${ }^{9}$ (The difference be-

\footnotetext{
* Electrochemical Society Active Member

${ }^{\mathrm{z}}$ E-mail: kawamura@spring8.or.jp
}

tween 13 and 12 was concluded to be within the error of the measurement.) However, the structural analysis using STM and AFM is still limited to the two-dimensional surface structure and is not able to clarify the structure normal to the surface, i.e., the threedimensional structure of the near-surface layers.

In the present study, the structures of the Te UPD layer formed on a $\mathrm{Au}(111)$ substrate have been investigated by using the in situ surface X-ray diffraction (XRD) technique. The structure normal to the substrate surface, which features the roughness and the composition of surface layers, can be investigated by specular X-ray reflectivity measurements. On the other hand, the two-dimensional periodicity of the atomic arrangement in the Te UPD layer is characterized by grazing incidence XRD measurements. Both such structural properties of the Te UPD layer are discussed, together with the comparison in the structural model reported in previous studies.

\section{Experimental}

The in situ surface XRD measurements were carried out using a к-type multiaxis diffractometer installed on beamline 14B1 at SPring-8, Japan. ${ }^{10}$ 14B1 delivers synchrotron radiation from a bending magnet, collimated by an Rh-coated mirror, monochromatized by a pair of $\mathrm{Si}(111)$ crystals, and focused onto the sample by another vertically bent mirror. The wavelength utilized was $\lambda=1.10 \AA$.

The geometry adopted for the measurements is shown in Fig. 1, where $\mathbf{k}_{\mathrm{i}}$ and $\mathbf{k}_{\mathrm{f}}$ correspond to the incident and the outgoing wave vectors, respectively. In order to describe the momentum transfer $\mathbf{Q}\left(=\mathbf{k}_{\mathrm{f}}-\mathbf{k}_{\mathrm{i}}\right)$ in terms of its components in the surface plane and along the surface normal direction, it is convenient to use a hexagonal coordinate system. The hexagonal unit cell is defined by $\mathbf{a}=a_{0}(1 / 2,0,-1 / 2)_{\text {cubic }}, \quad \mathbf{b}=a_{0}(-1 / 2,1 / 2,0)_{\text {cubic }}$, and $\mathbf{c}=a_{0}(1,1,1)_{\text {cubic }}$, where $a_{0}=4.079 \AA$. The reciprocal basis can be expressed as $\mathbf{a}^{*}=\left(2 \pi / a_{0}\right)(2 / 3,2 / 3,-4 / 3)_{\text {cubic }}$, $\mathbf{b}^{*}=\left(2 \pi / a_{0}\right)(-2 / 3,4 / 3,-2 / 3)_{\text {cubic }}, \quad$ and $\mathbf{c}^{*}=\left(2 \pi / a_{0}\right)$ $\times(1 / 3,1 / 3,1 / 3)_{\text {cubic }}$. An arbitrary position in hexagonal reciprocal space is represented by $(H, K, L)$ where $\mathbf{Q}=H \mathbf{a}^{*}+K \mathbf{b}^{*}$ $+L \mathbf{c}^{*}$. The relationship between $(h, k, l)_{\text {cubic }}$ and $(H, K, L)$ is given by the transformations: $h=(2 / 3) H-(2 / 3) K+(1 / 3) L, k$ $=(2 / 3) H+(4 / 3) K+(1 / 3) L, \quad$ and $\quad l=-(4 / 3) H-(2 / 3) K$ $+(1 / 3) L$. For example, $(1,1,1)_{\text {cubic }}=(0,0,3)$ and $(0,0,2)_{\text {cubic }}$ $=(-1,0,2)$. 


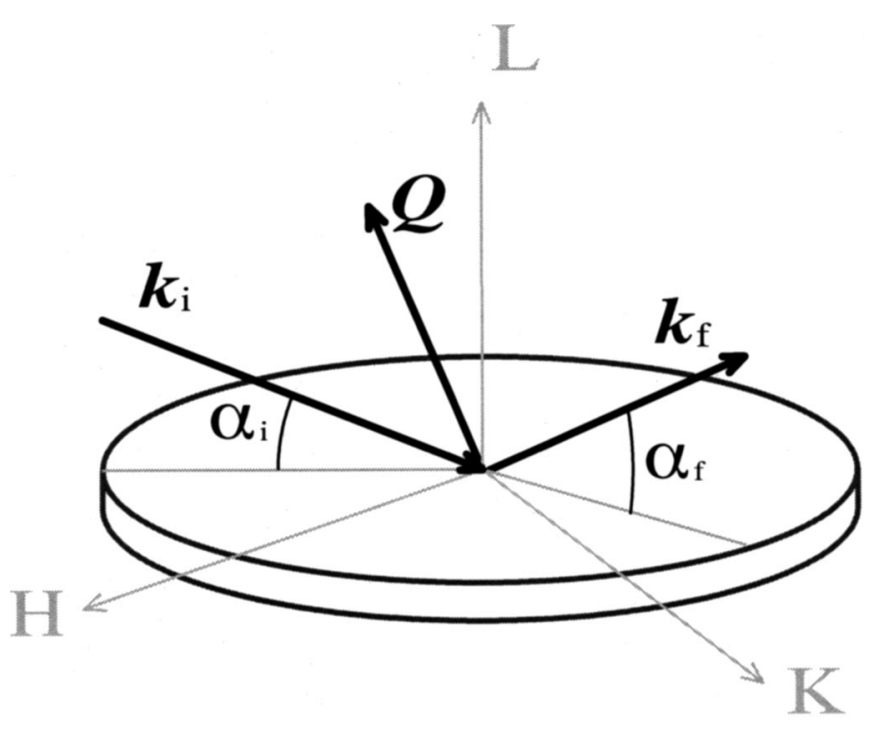

Figure 1. The experimental geometry of in situ surface XRD measurements.

The method to probe the reflectivity along $\mathbf{Q}=L \mathbf{c}^{*}$ with fixed $H$ and $K$ is called $\mathrm{X}$-ray reflectivity measurement, and provides the information about the interlayer spacings and the morphology of a sample. The method to measure the scattering intensity along $\mathbf{Q}$ $=H \mathbf{a}^{*}+K \mathbf{b}^{*}$ with $L \approx 0$ is called grazing incidence XRD and allows characterization of the two-dimensional periodicity of an atomic arrangement in a sample surface.

Specular X-ray reflectivity.-In a geometry where the incident and outgoing wave vectors ( $\mathbf{k}_{\mathrm{i}}$ and $\mathbf{k}_{\mathrm{f}}$, respectively) are in the same plane normal to the surface, and the incident angle $\alpha_{\mathrm{i}}$ and the outgoing angle $\alpha_{\mathrm{f}}$ are equal, the indexes $H$ and $K$ are equal to 0 . The $\mathrm{X}$-ray reflectivity in this geometry is called specular X-ray reflectivity.

Within the kinematical approximation, ${ }^{11}$ the theoretical specular reflectivity $R$ for the electrode surface is given by

$$
R=r_{\mathrm{e}}^{2} \lambda^{2} \frac{1}{\sin ^{2} \alpha} \frac{\left|F\left(Q_{z}\right)\right|^{2}}{\Gamma^{2}} \exp \left(-\frac{\mu}{\sin \alpha}\right)
$$

where $r_{\mathrm{e}}$ is the Thomson radius of the electron, $\Gamma$ is the area of the surface unit cell, $\alpha$ is the incident angle measured with respect to the sample surface, $\exp (-\mu / \sin \alpha)$ is the absorption correction due to the solution on the surface, and $Q_{z}=L\left|\mathbf{c}^{*}\right|$.

The structure factor $F\left(Q_{z}\right)$ is given by

$$
\begin{aligned}
F\left(Q_{z}\right)= & \sum_{m} \rho_{m} f_{m} \exp \left(-\frac{Q_{z}^{2} \sigma_{m}^{2}}{2}\right) \exp \left(i Q_{z} z_{m}\right) \\
& +f_{\mathrm{Au}} \exp \left(-\frac{Q_{z}^{2} \sigma_{\mathrm{Au}}^{2}}{2}\right) \sum_{n=0}^{\infty} \exp \left(i Q_{z} n d_{\mathrm{Au}}\right)
\end{aligned}
$$

The first term is the contribution from the surface layer $m$, and the second from the bulk $\mathrm{Au}(111)$. In the equation, $\rho_{m}$ is the coverage, $f_{m}$ is the atomic form factor, $z_{m}$ is the atomic position normal to the surface, and $\sigma_{m}$ is the root mean square (rms) of atomic displacement, including the effects of static corrugations and thermal vibrations. The values with a suffix Au correspond to the bulk of $\mathrm{Au}$, and $d_{\mathrm{Au}}$ is the interlayer spacing of $\mathrm{Au}(111)$.

In the quantitative determination of the structure of the nearsurface layers that include the surface adsorbate layers and the underlying Au layers, the parameters representing the electron density profiles, $\rho_{m}, f_{m}, z_{m}$, and $\sigma_{m}$ are optimized so as to adequately describe the observed reflectivity.
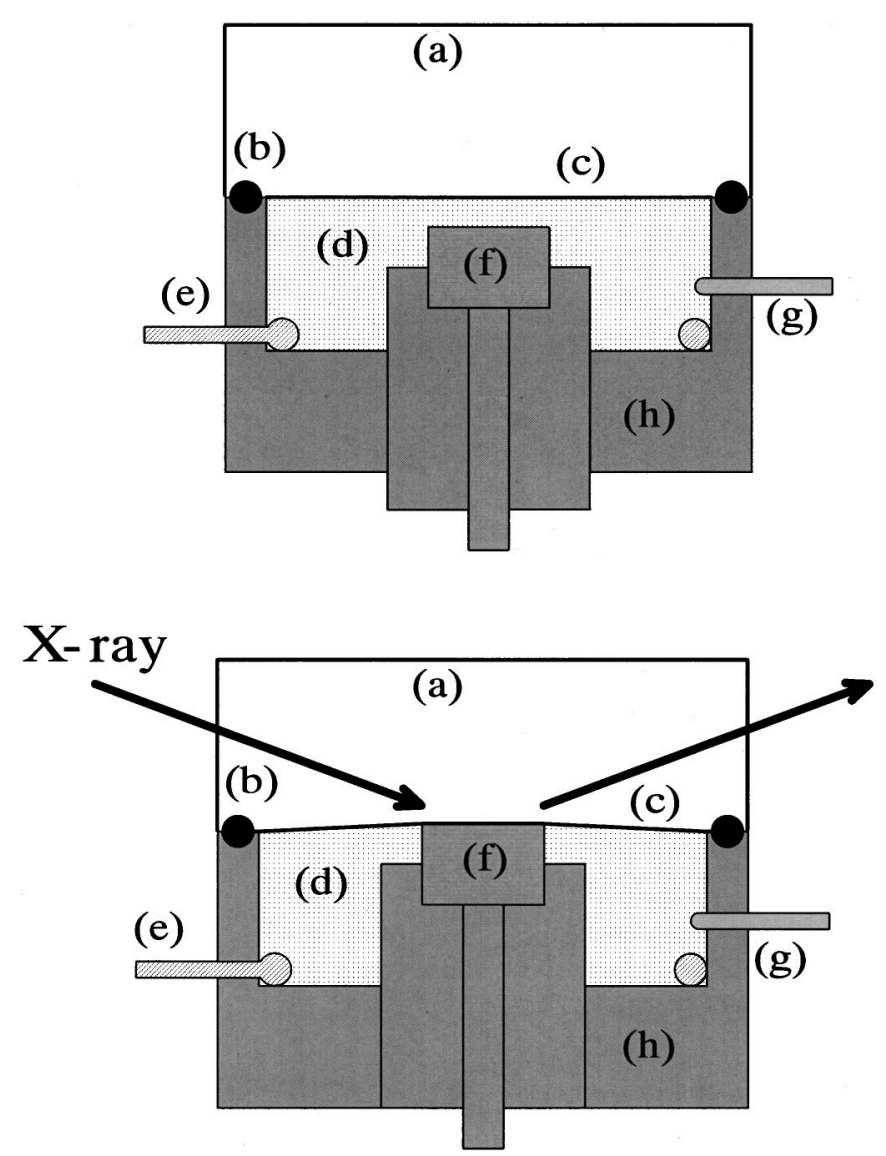

Figure 2. Electrochemical cell for the in situ surface XRD measurements, (top) during the electrochemical deposition and (bottom) during the in situ XRD measurement. (a) Mylar film chamber, (b) O-ring, (c) Mylar film, (d) electrolyte solution, (e) counter electrode, (f) $\mathrm{Au}$ (111) electrode, (g) reference electrode, and (h) PCTFE resin.

Grazing incidence XRD.-When the incident angle is held less than or in the order of the critical angle, the X-ray penetration depth is significantly reduced and the scattering from the interior of the sample is considerably decreased. In this geometry, the diffraction intensity along $\mathbf{Q}=H \mathbf{a}^{*}+K \mathbf{b}^{*}$ is probed with $L \approx 0$. The diffraction intensity is measured at the point where two-dimensional Bragg conditions are satisfied resulting from the periodicity of a unit cell of a surface layer, so that this measurement provides the information on the two-dimensional periodicity of the atomic arrangement in the surface layer.

Electrochemical conditions.-Commercial $\mathrm{Au}(111)$ disks (10 $\mathrm{mm}$ diam $\times 5 \mathrm{~mm}$ thick, MaTeck, Inc., Germany) were electrochemically polished at $c a .1 \mu \mathrm{A}$ for $6 \mathrm{~h}$ in a $0.1 \mathrm{M} \mathrm{HClO}_{4}$ $+5 \mathrm{mM} \mathrm{NaCl}$ solution, ${ }^{12}$ followed by an annealing in the air at $1123 \mathrm{~K}$ for $5 \mathrm{~h}$. Prior to each experiment, the disks were cleaned with concentrated sulfuric acid and annealed in a hydrogen flame. The Au(111) disk electrode was then transferred via air to the electrochemical cell and used as the working electrode. Counter and reference electrodes were made of Pt wire and an $\mathrm{Ag} / \mathrm{AgCl}$ (in 3.33 $\mathrm{M} \mathrm{NaCl}$ ) electrode, respectively. The electrolytic solution contained $0.1 \mathrm{mM} \mathrm{TeO}$ (Aldrich Chemical Corp., USA) and $10 \mathrm{mM} \mathrm{H}_{2} \mathrm{SO}_{4}$ (Kishida Chemical Co., Ltd., Japan) as the supporting electrolyte; the $\mathrm{pH}$ of the solution was 2.1 .

The electrochemical cell for the in situ surface XRD measurements was made of poly(chlorotrifluoroethylene) (PCTFE) resin as shown in Fig. 2. A $6 \mu \mathrm{m}$ thick Mylar film (Chemplex Industries, Inc., USA) was used as the X-ray windows. The electrochemical cell was connected to a glass vessel containing the electrolyte and to a 


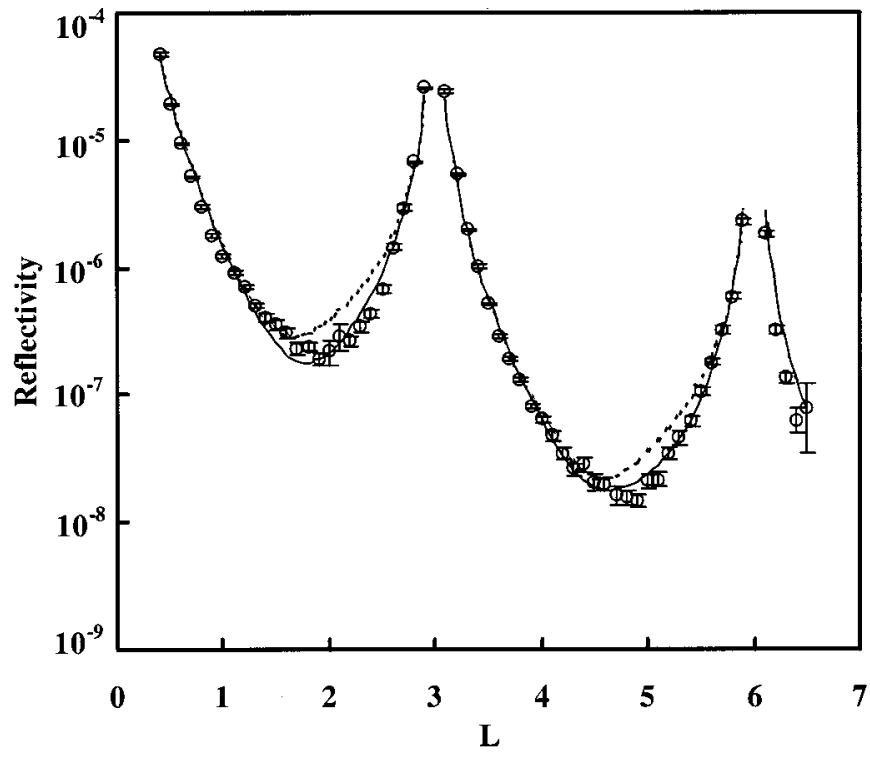

Figure 3. Specular X-ray reflectivity from the Te UPD layer on the Au(111) substrate. The data collection was started $4 \mathrm{~h}$ after the UPD potential was applied and lasted for $15 \mathrm{~h}$ in total. $(\bigcirc)$ Observed data. (----) The reflectivity expected for a Te layer immediately above the $\mathrm{Au}(111)$ substrate [structure parameters listed in Table I(a)]. ( - The reflectivity expected for the top layer consisting of $0.33 \mathrm{ML}$ Te and $0.02 \mathrm{ML} \mathrm{Au}$ [structure parameters listed in Table $\mathrm{I}(\mathrm{b})]$.

cylinder of an ultrapure $\mathrm{N}_{2}$ gas using PCTFE tubes and cocks. After deaeration of the electrolyte, the cell was purged with $\mathrm{N}_{2}$ gas, and then the electrolyte was slowly transferred into the cell through the tube by applying a mild pressure of $\mathrm{N}_{2}$ gas; this allows transfer of the electrolyte without exposing it to air. The potential of $0.80 \mathrm{~V}$ was applied to the working $\mathrm{Au}(111)$ electrode during the injection of the electrolyte into the cell. The thickness of the electrolyte layer between the $\mathrm{Au}(111)$ surface and the Mylar film was in the millimeter range. Then the potential was lowered to $0.20 \mathrm{~V}$ with a scan rate of $10 \mathrm{mV} \mathrm{s}^{-1}$ and was kept at $0.20 \mathrm{~V}$ for $30 \mathrm{~min}$ to complete the UPD of Te. The time required for completion was determined by the preliminary deposition-stripping experiments. Then the potential was brought back in the Te UPD region to $0.23 \mathrm{~V}$ with a scan rate of $10 \mathrm{mV} \mathrm{s}^{-1}$ in order to avoid further deposition of Te, because 0.20 $\mathrm{V}$ is equal to the equilibrium potential of bulk Te deposition calculated from thermodynamic data. Immediately before the in situ sur- face XRD measurement, the $\mathrm{Au}(111)$ electrode was moved close to the Mylar film so that the electrolyte layer was as thin as $10 \mu \mathrm{m}$.

The electrochemical cell for the voltammetric study, using the hanging meniscus method, was made of glass tube. After deaeration of the electrolyte, the $\mathrm{Au}(111)$ electrode which was mounted on an electrode holder was dipped into the solution. When the electrode was in contact with the solution, the potential was $0.80 \mathrm{~V}$, that is more positive than both the deposition and stripping potentials of Te. Voltammograms were obtained by scanning the potential at a scan rate of $10 \mathrm{mV} \mathrm{s}^{-1}$. The cathodic potential limit was $-0.20 \mathrm{~V}$.

\section{Results}

Specular X-ray reflectivity measurements.-The specular reflectivity for the Te UPD layer formed on a $\mathrm{Au}(111)$ substrate is shown in Fig. 3. The rapid increases in the intensity at $L=3$ and 6 correspond to the $(1,1,1)_{\text {cubic }}$ and $(2,2,2)_{\text {cubic }}$ Bragg reflections, respectively. Since the alignment of the diffractometer took several hours, the data collection started $4 \mathrm{~h}$ after the UPD potential was applied and lasted for $15 \mathrm{~h}$ in total. The dotted line is the calculated profile based on a model which assumes that the interlayer spacings of layers near the surface are the same as the interlayer spacings of the bulk $\mathrm{Au}(111)$ planes, and that the coverage of Te is 0.33 monolayer (ML). The structure parameter used to construct the profile are listed in Table $\mathrm{I}(\mathrm{a})$, and the model has a fairness of $R$ factor, $R$ $=12.8 \%$. The observed reflectivity is also compared to the calculated reflectivity (solid line in Fig. 3) based on the structure parameters listed in Table I(b). Here, the first layer consists of $0.33 \mathrm{ML}$ Te and 0.02 ML Au, while the second layer consists of 0.98 ML Au. This model provides a slightly better description of the data $(R$ $=12.3 \%$ ), and the calculated profile apparently better describes the left side of the Bragg peaks in Fig. 3 than that of the former model.

The structure of the Te UPD layer formed on the $\mathrm{Au}(111)$ substrate was found to change with time. Figure 4 shows the specular reflectivity measurement repeated on the same sample. The data collection in this case started $39 \mathrm{~h}$ after the UPD potential was applied and lasted for $20 \mathrm{~h}$ in total. The profiles calculated from the structure parameters listed in Table I(b) (dotted line in Fig. 4), however, do not describe the observed reflectivity (in this case, $R$ $=30.9 \%)$. The solid line is another reflectivity profile calculated from the structure parameters listed in Table I(c). This model produced better fit $(R=14.3 \%)$ than the model based on the parameters listed in Table $\mathrm{I}(\mathrm{b})$. Here, the first layer consists of $0.33 \mathrm{ML} \mathrm{Te}$ and 0.08 ML Au, while the second layer consists of 0.92 ML Au. The Te UPD layer is no longer on the ideally terminated $\mathrm{Au}(111)$ surface.

\section{Table I. List of the structure parameters to describe the observed reflectivity. ${ }^{\text {a }}$}

1st layer

$\mathrm{Te}$

(a) Model for the top layer consisting of $0.33 \mathrm{ML}$

Coverage $\rho$

rms of displacement $\sigma(\AA)$

Position $z(\AA)$

\section{$0.33^{\mathrm{b}}$}

$0.085^{\mathrm{b}}$

$-2.355^{\mathrm{b}}$ 2nd layer

$\mathrm{Au}$

$1.00^{\mathrm{b}}$

$0.085^{\mathrm{b}}$

$0.00^{\mathrm{b}}$ $n$th layer $(n>2)$

$\mathrm{Au}$

(b) Model for the top layer consisting of 0.33 ML Te and 0.02 ML Au

Coverage $\rho$

rms of displacement $\sigma(\AA)$

$$
0.33^{\mathrm{b}}
$$

$0.20 \pm 0.05$

Position $z(\AA)$

$-2.12 \pm 0.02$

$$
\begin{array}{r}
0.02 \pm 0.02 \\
0.20 \pm 0.10 \\
-2.12 \pm 0.19
\end{array}
$$

$0.08 \pm 0.01$
$0.30 \pm 0.20$
$-2.40 \pm 0.02$
$0.98 \pm 0.01$

$0.19 \pm 0.02$

$0.00 \pm 0.02$

$0.92 \pm 0.01$

$0.22 \pm 0.02$

$-0.09 \pm 0.01$ $1.00^{\mathrm{b}}$

$0.085^{\mathrm{b}}$

$2.355 \times(n-2)^{\mathrm{b}}$

$1.00^{\mathrm{b}}$

$0.085^{\mathrm{b}}$

$2.355 \times(n-2)^{\mathrm{b}}$

$1.00^{\mathrm{b}}$

$0.085^{\mathrm{b}}$

$2.355 \times(n-2)^{\mathrm{b}}$

(c) Model for the top layer consisting of $0.33 \mathrm{ML}$ Te and 0.08 ML Au

rms of displacement $\sigma(\AA)$

$0.20 \pm 0.10$

Position $z(\AA)$

$-2.40 \pm 0.01$

${ }^{\text {a }}$ Errors obtained by changing the parameters to keep the $R$ factor deviation within $0.1 \%$.

b Parameters that are held fixed.
Downloaded on 2012-09-26 to IP 133.87.26.18 address. Redistribution subject to ECS license or copyright; see www.esltbd.org 


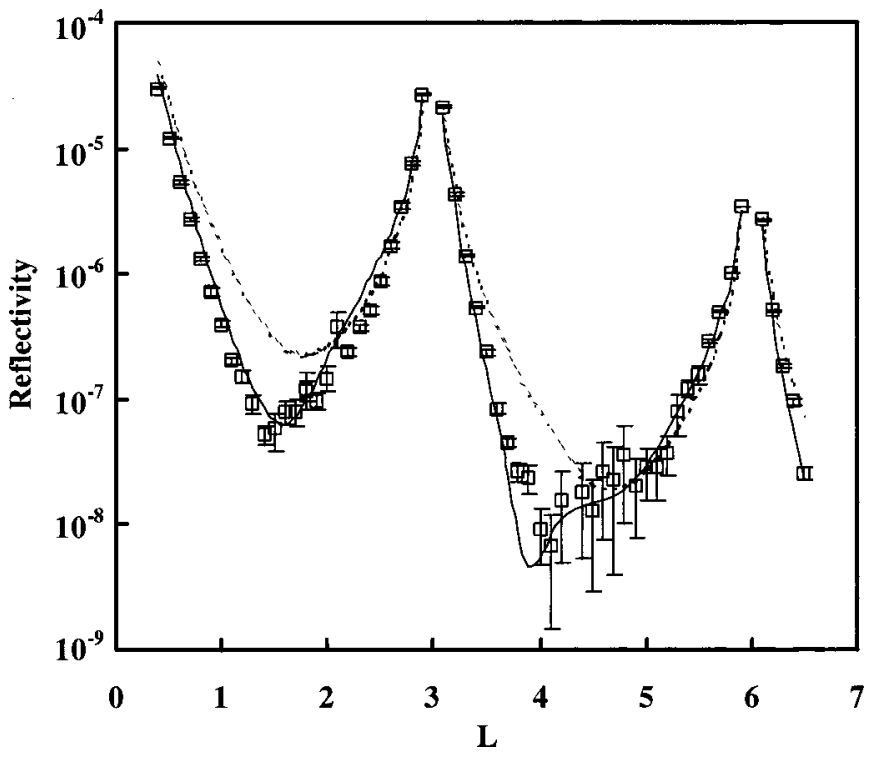

Figure 4. Specular X-ray reflectivity from the Te UPD layer on the Au(111) substrate. The data collection was started $39 \mathrm{~h}$ after the UPD potential had been applied and lasted for $20 \mathrm{~h}$ in total. ( $\square$ ) Observed data. (----) The reflectivity expected for the top layer consisting of $0.33 \mathrm{ML}$ Te and $0.02 \mathrm{ML}$ $\mathrm{Au}$ [structure parameters listed in Table I (b)]. ( - The reflectivity expected for the top layer consisting of 0.33 ML Te and 0.08 ML Au [structure parameters listed in Table I(c)].

Grazing incidence XRD measurements.-After the specular reflectivity was measured as mentioned above, the grazing incidence diffraction measurement was carried out in a geometry where $L$ $=0.2$. Figure 5 shows the measured scattering intensity acquired by carrying out scans along the $\mathbf{Q}=x \mathbf{a}^{*}+x \mathbf{b}^{*}$ direction $(x$ $=0.15-0.75)$. When the potential is maintained at $0.23 \mathrm{~V}$, four peaks appear at $x=0.26,0.40,0.65$, and 0.68 . The peaks at $x$ $=0.26,0.40$, and 0.68 are due to the Mylar film used as the X-ray windows of the electrochemical cell, which was confirmed in the other grazing incidence XRD measurements. On the other hand, when the potential is changed from 0.23 to $0.80 \mathrm{~V}$ to strip the Te

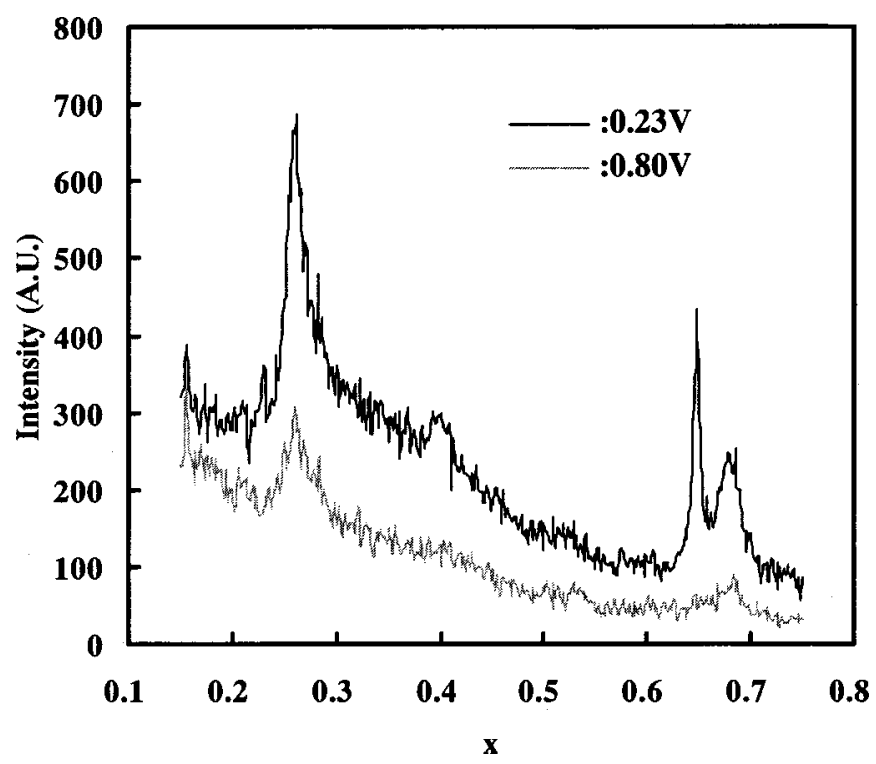

Figure 5. Grazing incidence XRD scans for $\mathrm{Au}(111)$ surface in a solution of $0.1 \mathrm{mM} \mathrm{TeO}_{2}+10 \mathrm{mM} \mathrm{H}_{2} \mathrm{SO}_{4}$ while the potential was maintained at 0.23 or $0.80 \mathrm{~V}$. The background was not subtracted.

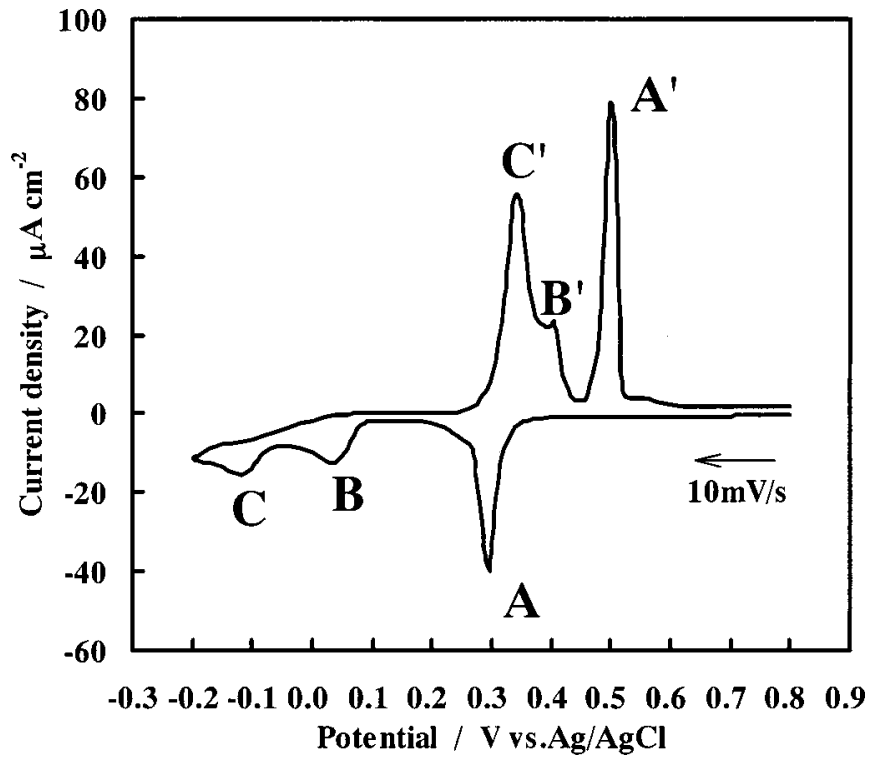

Figure 6. Cyclic voltammogram for the $\mathrm{Au}(111)$ electrode in a solution of $0.1 \mathrm{mM} \mathrm{TeO}{ }_{2}+10 \mathrm{mM} \mathrm{H}_{2} \mathrm{SO}_{4}$. The peak pair $\mathrm{A} / \mathrm{A}^{\prime}$ corresponds to the first Te UPD and the corresponding dissolution, $\mathrm{B} / \mathrm{B}^{\prime}$ to the second Te UPD and the corresponding dissolution, and $\mathrm{C} / \mathrm{C}^{\prime}$ to the bulk Te deposition and the corresponding dissolution.

UPD layer, the peak at $x=0.65$ clearly disappears. Therefore, it is concluded that the scattering intensity which appears at $x=0.65$ originates from the Te UPD layer. The Te UPD layer does not have the $(\sqrt{3} \times \sqrt{3}) \mathrm{R} 30^{\circ}$ structure, which is confirmed in that no diffraction intensity is observed at $x=0.33$, but has an another structure. We have examined several in-plane orientations, and the intensity found at $x=0.65$ was not observed at all in-plane orientations. Thus, the intensity found at $x=0.65$ is not due to a polycrystalline structure. This structure is discussed in the following section.

Electrochemical measurements.-Figure 6 shows the cyclic voltammogram obtained for the $\mathrm{Au}(111)$ electrode in a solution of 0.1 $\mathrm{mM} \mathrm{TeO}$ and $10 \mathrm{mM} \mathrm{H}_{2} \mathrm{SO}_{4}$, using the hanging meniscus method. Three cathodic peaks due to the deposition of a first UPD, a second UPD, and bulk Te appeared at (A) 0.30, (B) 0.04, and (C) $-0.11 \mathrm{~V}$, respectively, while the corresponding anodic peaks were recognized at $\left(\mathrm{A}^{\prime}\right)$ 0.35, $\left(\mathrm{B}^{\prime}\right) 0.40$, and $\left(\mathrm{C}^{\prime}\right) 0.50 \mathrm{~V}$. These are in good agreement with the literature, ${ }^{6-8}$ suggesting that the UPD behavior of Te on a $\mathrm{Au}(111)$ surface is identical to those already reported.

Figure 7 shows the stripping voltammograms for Te UPD layers formed on a $\mathrm{Au}(111)$ surface after holding the potential at $0.23 \mathrm{~V}$ for 0.5 to $12.1 \mathrm{~h}$; here, the potential was swept from 0.23 to $0.80 \mathrm{~V}$ with the scan rate of $10 \mathrm{mV} \mathrm{s}^{-1}$. An anodic peak corresponding to the stripping of Te layer, i.e., peak $\mathrm{A}^{\prime}$, was observed at 0.51-0.52 V. On the other hand, another shoulder peak was identified at $0.53 \mathrm{~V}$ for samples with longer holding time, and the peak current at $0.53 \mathrm{~V}$ increased with longer holding time, while the peak at $0.50 \mathrm{~V}$ decreased.

The total charge for the stripping of the Te UPD layer was estimated from the peak area, assuming that the associated charge is related only to the following four-electron reaction

$$
\mathrm{Te}+2 \mathrm{H}_{2} \mathrm{O} \rightarrow \mathrm{HTeO}_{2}^{+}+3 \mathrm{H}^{+}+4 \mathrm{e}^{-}
$$

The charge was $0.27 \mathrm{ML}(0.5 \mathrm{~h}), 0.28 \mathrm{ML}(1.7 \mathrm{~h}), 0.33 \mathrm{ML}(7.3 \mathrm{~h})$, and $0.32 \mathrm{ML}(12.1 \mathrm{~h})$, respectively. It turned out that the stripping charge saturated at the coverage of $0.33 \mathrm{ML}$ after a holding time of $7.3 \mathrm{~h}$, despite the variations observed in the stripping current profile. 


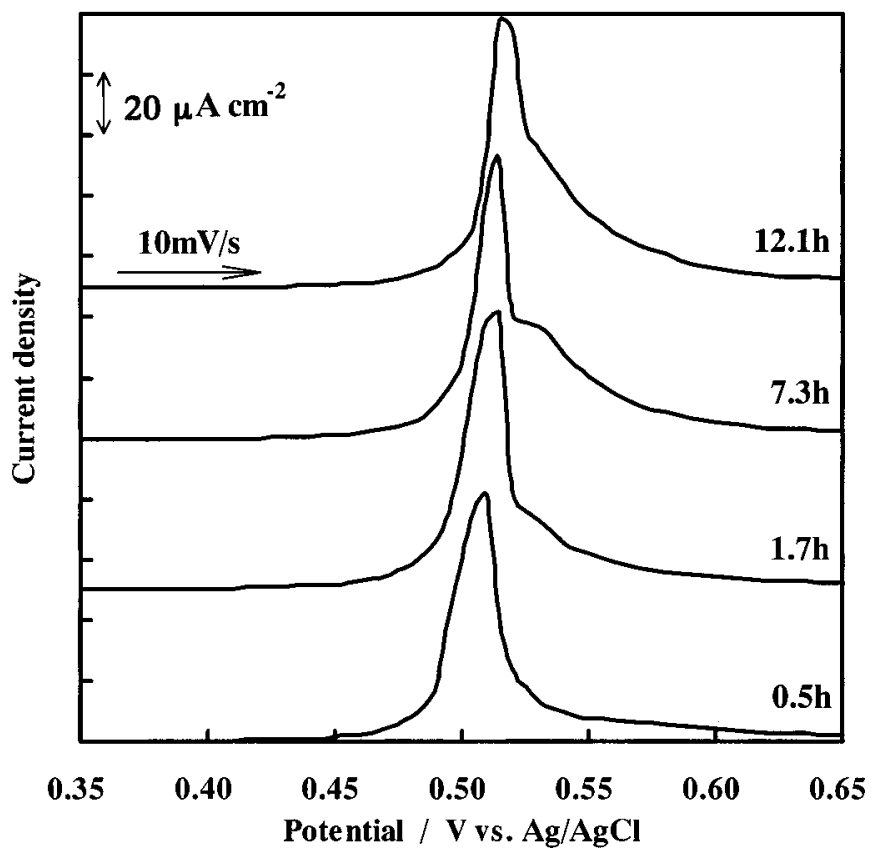

Figure 7. Stripping voltammograms for Te UPD layer formed on a Au(111) electrode after holding the potential at $0.23 \mathrm{~V}$ for $0.5,1.7,7.3$, and $12.1 \mathrm{~h}$.

The result for the coverage is in good agreement with the literature ${ }^{7,8}$ and supports the Te coverage of 0.33 ML given by the theoretical reflectivity calculation.

\section{Discussion}

The X-ray reflectivity measurement indicates that the structure of the Te UPD layer on the Au(111) substrate is changing with time. The structure parameters listed in Table I clearly demonstrate that a portion of $\mathrm{Au}$ atoms migrate from the top layer of the $\mathrm{Au}(111)$ substrate to the topmost Te layer. As a result, the first layer consists of both $\mathrm{Te}$ and $\mathrm{Au}$ atoms. The corrugation of the first and second layer also increases with time.

An STM study showed that the diffusion of Au into the Te UPD layer appeared as a pitted surface for the Te second UPD layer but did not appear for the Te first UPD layer. ${ }^{9}$ Although the applied potential was different, the possibility for the diffusion of $\mathrm{Au}$ is suggested in the Te UPD layer. ${ }^{9}$ It is unclear whether the pitted surface forms in the Te UPD layer; however, the surface change for the Te UPD layer may be observed in STM and AFM observations after sufficient time has passed, because the diffusion of $\mathrm{Au}$ is a kinetically slow process.

The grazing incidence XRD measurements show that after ample time elapses, the Te UPD layer does not have a periodicity such as $(\sqrt{3} \times \sqrt{3}) \mathrm{R}^{\circ} 0^{\circ}(\mathrm{Te}-\mathrm{Te}$ spacing of $4.995 \AA)$, but another regular structure in the top layer, although the top layer consists of both Te and $\mathrm{Au}$ atoms. Bulk Te consists of a series of chains of Te atoms (Te-Te distance of $2.84 \AA$ and Te-Te-Te angle of $103^{\circ}$ ) and has a hexagonal structure $(a=4.458 \AA, c=5.927 \AA)$. If the Te UPD layer with a small amount of $\mathrm{Au}$ atoms has a hexagonal structure with the atomic spacing of $4.458 \AA$, the diffraction intensity must be observed at $x=0.647$, which is almost equal to the observed one. Also, if the Te UPD layer has the hexagonal structure with the atomic spacing of $4.458 \AA$, the coverage of the Te UPD layer is $0.42 \mathrm{ML}$. The structure parameters listed in Table I shows that the Te UPD layer consists of $0.33 \mathrm{ML}$ Te and $0.08 \mathrm{ML} \mathrm{Au}$, and the total coverage becomes $0.41 \mathrm{ML}$, which is almost equal to that of the hexagonal structure. Then the hexagonal structure similar to bulk Te might exist in the Te UPD layer, but the results in this study are still inconclusive, and further investigation is needed in order to precisely describe the structure of the Te UPD layer.
The present study shows that the Te UPD layer does not have a regular structure such as $(\sqrt{3} \times \sqrt{3}) \mathrm{R} 30^{\circ}$ but has an another structure by the diffusion of Au into the Te UPD layer after ample time elapses. The original structure of the Te UPD layer may be demonstrated by grazing incidence XRD measurements for the as-formed Te UPD layer, if possible.

The structural change in the Te UPD layer is also suggested by the electrochemical measurements. In stripping voltammetry, the peak of the anodic dissolution of Te UPD layer at $c a$. $0.51 \mathrm{~V}$ shifts positively with longer time for holding the potential at which $\mathrm{Te}$ UPD takes place. The increase of another anodic peak at $0.53 \mathrm{~V}$ as a shoulder indicates that a strong interaction of Te atoms in the $\mathrm{Te}$ UPD layer with $\mathrm{Au}$ atoms in the substrate occurs with time.

As mentioned in the introduction, deposition of $\mathrm{Cd}$ on a gold surface leads to the formation of a $\mathrm{Cd}-\mathrm{Au}$ alloy, ${ }^{3}$ which induces a structural change of the gold surface. Therefore, Te, which is less reactive with $\mathrm{Au}$, is deposited first in the ECALE studies to form CdTe bilayers. According to the phase diagrams of the Te-Au system, ${ }^{13}$ however, it is possible that gold and tellurium form a $\mathrm{Te}-\mathrm{Au}$ alloy, such as a calaverite, an important source of gold in several areas of the world. ${ }^{14}$

Consequently, an ideal single atomic layer formed by the UPD process is not always stable for a long period of time. In a UPD process, atomic diffusion may occur within the near-surface layers. Should this be the case, the top layer structure differentiates from that expected based on the STM and AFM observations which are typically completed within $1 \mathrm{~h}$ or less. Since the XRD analysis of thick deposits of CdTe by ECALE shows the formation of cubic CdTe with the (111) preferential orientation, ${ }^{4}$ an ideally atomic layer just on the substrate is not always necessary for the formation of thick deposits using the ECALE method, in which the possible interactions with the underlying substrate become negligible. However, the interaction with the substrate becomes considerable in the formation of very thin deposits using ECALE, especially in the formation of a few atomic layers to produce some applications consisting of ultrathin layers.

\section{Conclusion}

The in situ surface XRD technique revealed that the interlayer structure of the Te UPD layer on the Au(111) substrate could not be described by the simple structure such that the Te layer was formed just on the $\mathrm{Au}(111)$ substrate. The top layer consists of the Te atoms and $\mathrm{Au}$ atoms that moved from the $\mathrm{Au}(111)$ substrate. In addition, the top and second layers are considerably corrugated. The Te UPD layer does not have a regular structure such as $(\sqrt{3} \times \sqrt{3}) \mathrm{R} 30^{\circ}$ but has another structure after ample time elapses. The change of the Te UPD layer with time is also suggested by the stripping potential shift in the stripping voltammograms.

\section{Acknowledgment}

The present study was partly supported by Grants-in-Aid for Scientific Research (no. 11750713) from the Japan Society for the Promotion of Science.

The Synchrotron Radiation Research Center, Japan Atomic Energy Research Institute, assisted in meeting the publication costs of this article.

\section{References}

1. J. L. Stickney, in Electroanalytical Chemistry, Vol. 21, A. J. Bard and I. Reubenstein, Editors, p. 75, Marcel Dekker, New York (1999), and references cited therein.

2. B. W. Gregory, D. W. Suggs, and J. L. Stickney, J. Electrochem. Soc., 138, 1279 (1991).

3. B. E. Hayden, and I. S. Nandhakumar, J. Phys. Chem., 102, 4897 (1998).

4. L. P. Colletti, B. H. Flowers, Jr., and J. L. Stickney, J. Electrochem. Soc., 145, 1442 (1998).

5. J. W. Schulze, F. D. Koppitz, and M. M. Lohrengel, Ber. Bunseng-Ges. Phys. Chem., 78, 693 (1974).

6. B. E. Hayden and I. S. Nandhakumar, J. Phys. Chem., 101, 7751 (1997).

7. D. W. Suggs and J. L. Stickney, J. Phys. Chem., 95, 10056 (1991). 
8. N. Ikemiya, D. Iwai, K. Yamada, R. Vidu, and S. Hara, Surf. Sci., 369, 199 (1996)

9. T. A. Sorenson, K. Varazo, D. W. Suggs, and J. L. Stickney, Surf. Sci., 470, 197 (2001).

10. M. Takahasi and J. Mizuki, J. Synchrotron Radiat., 5, 893 (1998).

11. D. Gibbs, B. M. Ocko, D. M. Zehner, and S. G. J. Mochrie, Phys. Rev. B, 38, 7303 (1988).
12. S. Ye, C. Ishibashi, K. Shimizu, and K. Uosaki, J. Electrochem. Soc., 145, 1614 (1998).

13. T. B. Massalski, H. Okamoto, P. R. Subramanian, and L. Kacprzak, Binary Alloy Phase Diagrams, 2nd ed., Vol. 1, p. 441, ASM International, Metals Park, OH (1996).

14. S. Jayasekera, J. Avraamides, and I. M. Ritchie, Electrochim. Acta, 41, 879 (1996). 